\title{
A Study on Setting up a Database for Maternal Health Care Program in Darbandikhan-Kurdistan*
}

\author{
Jamal K. Shakor, Sabah Sh. Mohammed, Yadgar H. Hamakarim, and Abbas M. Ahmed \\ Darbandikhan Technical Institute \\ Sulaimani Polytechnic University
}

\begin{abstract}
There are around 8.64 million women in Iraq who need to maternal health. While, maternal health program in Iraq is considerably down regulated, the vertical national health program, maternal health, has been not monitored appropriately. The aim of this study was to determine the most essential preliminary indicators for monitoring maternal health program in Kurdistan/ Iraq, and to assess those technical challenges for computerizing the maternal health program of Kurdistan Regional Government. This is interventional hospital based study which was carried out in Darbandikhan district- Kurdistan State. An access database was developed for entering whole records of the maternal health program components data in the 200 mothers' file. And, we got staff belief about database. The study has shown that antenatal care visit record would be a good indicator for assessing maternal health program, when the program was computerized. And, the staff had a positive attitude to computerize the program. This database could accelerate the monitoring and evaluating the maternal health program in considering to indicators of the program.
\end{abstract}

Key words: Database, Maternal Health Care Program, Program Indicators.

\section{Abbreviations: \\ PHCC: primary health care center \\ TBA: traditional birth attendant \\ EDD: expect date of delivery \\ MDG: millennium development goal}

\section{Introduction}

Women in reproductive age (15-49 year) consist of almost one-fourth of total Iraqi population who is considered to be 8.64 million women in 2015 [1]. Iraqi health system has to provide maternal health care to this group in the package of primary health care [2]. Maternal health refers to the health of women during pregnancy, childbirth, and the postpartum period [3]. In similar concerning, health care during pregnancy in Iraq, which include both preventive care (such as antenatal care, growth monitoring and immunization), and curative services (such as treatment of ailments) are provided for the women in health care centers, whereas labor and post natal care, with some investigations are provided for the women in general or private hospital [4], [5].

Furthermore, a survey study has shown which, antenatal care is delivered by PHCC (32.4\%), public hospital (19\%), and private doctors (44\%) [2]. Despite of this health system, Iraq has launched a national program for maternal health.

Maternal health was confirmed in fifth goal of Millennium Developing Goal (MDG), and most of the countries have launched a program to achieve the objectives of MDG [6]. Maternal health program in Iraqi health strategies 2008 has integrated with other programs such as child and reproductive health program. The main components of maternal health goal in these strategies are skilled attendant at birth for all pregnant women, improvement of health status of women, maternal morbidity and mortality surveillance system, supervision of TBAs performance, development appropriate strategic polices for reproductive health including antenatal, postnatal, family planning, and emergency obstetric cares.

Depending on the components of the strategies, maternal health program in Iraq is considerably down regulated. Maternal mortality in Iraq is high comparatively which is estimates very wide from 63 per 100000 live births [7] to 25 per 100000 live births [8]. Furthermore, maternal mortality contributed $3 \%$ of total mortality of reproductive age females (15-49 years) in Iraq in 2012. In the same concerning, it was reported that $16.5 \%$ of death in the same age group in the hospitals was due to maternal causes in the country [9].

Antenatal care coverage in Iraq still doesn't meet the strategic plan compared with developing countries. It has been reported in developing countries nearly $70 \%$ of pregnant women have at least one antenatal care visit, but in Iraq first antenatal care visit is higher than this rate. However in Iraq, antenatal care coverage which is recommended (four visit and above) is comparatively low to developing countries [10]. Antenatal care coverage in Iraq is still low (29\%), and similarly for postnatal coverage (41\%) [11]. From another side, in Iraq around three quarters of all births occurs outside the health-care institutions until 2014, yet over a fifth $(22 \%)$ of deliveries is classed as high risk and in need of advanced medical support. 
A study has shown that Iraqi health system faced many challenges, poor information technology and poor health authorities have determined as the main challenges of primary health care [12].

For instance, maternal health program is annually monitored and evaluated only through some general indicators such as, maternal mortality ratio, antenatal care coverage, skilled attendant at birth, and postnatal care for mothers and babies, whereas these monitoring and evaluation are paper-based, and are conducted through some local surveys which mostly all surveys have been conducted by WHO or UNICEF [13]. Regarding to preserving data, a study has reported that many mistakes data in the hospitals have been recorded about maternal mortality and as well as system of clear data entry has recommended in the same study [9]. From another side, another study on view of women toward maternal and antenatal care has reported that the poor utilization of public services has determined as main challenge to women to not prefer public sector instead of using of private sector [14]. Due to all above challenges remain in Iraqi health system could not monitor the vertical national health programs such as maternal health.

To achieve right data for monitoring and evaluating the goals of maternal health care program, this study has developed a database in aim to determine the most essential preliminary indicators for maternal health program in Kurdistan/ Iraq, and to determine those technical challenges for data-basing the maternal health program of Kurdistan Regional Government.

\section{Methodology}

This is interventional hospital based study which was conducted in Darbandikhan district- Kurdistan StateIraq. More than 13000 mothers have attained maternal health care in Darbandikhan health center during each antenatal and postnatal care visit. In the first visit when the staff has to produce a new file to all mother information which encompasses the demographic, family, medical and obstetric histories, immunization, the risk associated with pregnancy, physical and laboratory examinations, and ultrasound. Then, under maternal health program each pregnant woman depends on program recommendations has to regularly visit the health center. Thus, eleven visits during antenatal care and five visits during postnatal care have been recommended. In each visit, physical examinations such as measurement of weight and blood pressure, as well as in each alternative visit laboratory examinations such as blood group, HB, blood sugar, general urine examination, albumin urea, and general stool examination are conducted for each pregnant woman. These all information will be recorded in the mother's files. As a part of this service, all data regarding to maternal health have been collected, recorded and archived in the health center in a file form. Each mother has a code of family number which indicates mother's data in the file. All files depending on this code have been organized. In each antenatal and postnatal visit, the new data regarding to maternal health for each pregnant women will be added to the file. This study recruited 200 mother's file randomly to the database from the health center for the research project.

\section{The Database}

Database has designed base on maternal health program of Ministry of Health- KRG. An access database 2010 has been developed for entering whole recorded data in the mother's file that have recommended depends on maternal health program. All components of the mother's file such as demographic, medical, family, obstetric histories, immunization, risk factors associated with pregnancy, physical examination, laboratory examination, and postnatal have formed the database. In the database, one table was made for each component, and accordingly more than 300 fields in the tables were made for whole variables in mother's file. Database almost included all the variables and it has been established in such way which could determine the main indicates of maternal health program. In order to examine the database, previously few files were entered into the database and revised many time until last version which was feasible and applicable to use.

Four queries were formed for the main indicators which were used for evaluating the maternal health program. The first query was created for antenatal visits, the query describes rate of women who were covered. The covered women defined as the women who visited health center four times or more during antenatal care, (Covered women in specific period $=$ Women complete 40 weeks (group of women who meet EDD) - Women had < 4 visits). In addition, for analysis purpose the covered rate was found out.

Second query was designed for postnatal visits and the query defined woman as coverage if the woman had been visited one time or more during postnatal care. Third query was developed to determine the delivery attending skilled personal or not. And last query was assigned to define maternal mortality (how many mothers during antenatal and postnatal period died to 100000 live births?)

\section{Intervention}

The database has been introduced to the staff in a workshop, and they have been trained about data entering. Then, they were participated in data entry. Data entry process lasted for 30 days and the process was under observation. Participants were consisted of 
five special nurses of the maternal health program and another three stakeholders who were in-charge of the program. In the last day they have been interviewed based on preexisting questioned interview schedule. The participants were asked about feasibility, and obstacles associated with using of the database for the program.

\section{Tools and measurement}

This study has used three different methods for collecting data; using database for analyzing data, observation, and interview. Analyzing data by using database have been used to determine the indicators which are assessed the maternal health program.

Observation has been taken for assessing the technical problems that may associate with data entering process into the computer. Time required for entering one file to the database was observed after one day of training. Five different staff participated in entering data, each of them entered two files to the database, and required time for entering each file was recorded, and the required time for entering one file was calculated based on average of all recorded time. Another observation was to measure the number of mother who visits this department (maternal care) in the days. In this instance, we looked at their record for two weeks, and got average visiting number in days for analyzing purpose. Two types of visit were measured, first visit when the staff ought to make new file to the mother and keep her file by new code. All information has to save in this file. Then under maternal health program mothers have to regularly visit department for maternal health care. This all information will be added to the mother's files.

Interview with staff have been used for addressing the logistic problems which may be associated with process of computerizing the program. The interview was based preexisting open end question which was measured their opinion and belief about the implementation of the database program. Those questions encompass 1) to which extend this database is feasible to maternal health program? 2) How could this database help the program of maternal health? 3) What will be the problems when maternal health program have been used this database? 4) What could be added to this database or removed?

\section{Results}

Two hundred of mothers' files were entered to the database. This database could provide ongoing report about the main four indicators of maternal health of Iraqi governments, but this study found that antenatal visits and attending health institutions for delivery were the main indicators for the program. Ongoing reports about those indicators were illustrated in this study. First indicator about antenatal visits showed in figure 1. In this report, the database showed that, between $01 / 01 / 2014$ to $01 / 10 / 2014,91 \%$ of women has covered (rate women who have four or more visit to health center during antenatal care).
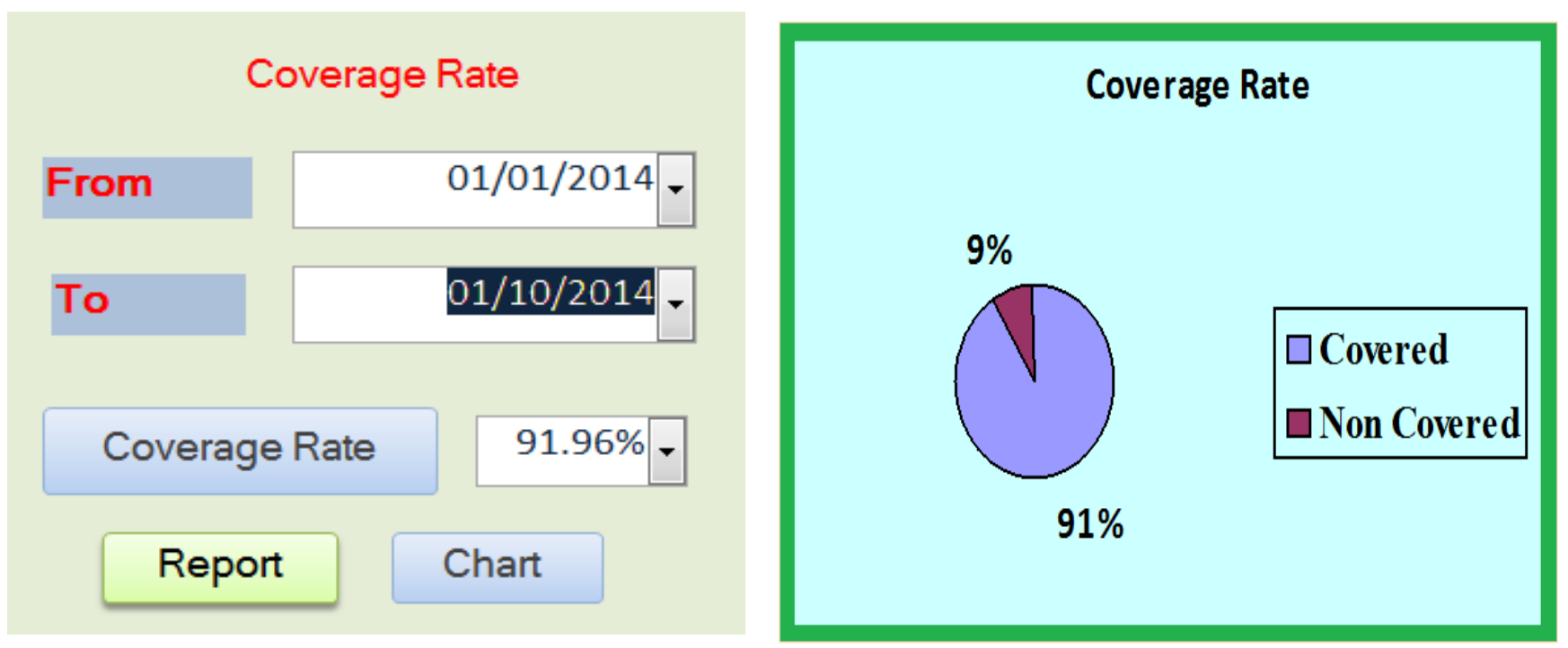

Figure 1: Report about antenatal visits. 
From

$01 / 01 / 2013$

To

\section{Chart Report}

\section{Placee of delivery}

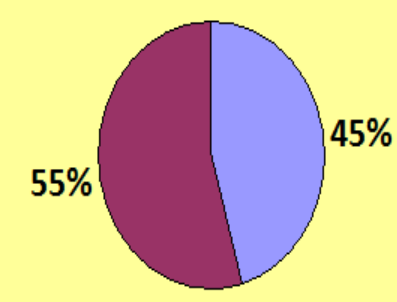

\section{$\square$ Domestic}

$\square$ Health Institutions

Figure 2: Report about rate of labor at health institutions.

Another report was about rate of women who attended health institutions for delivery. In this report, figure 2, database displayed, during one year and six months, between $01 / 01 / 2013$ to $01 / 06 / 2014,55 \%$ of women attended health institutions for delivery. And the rest (45\%) preferred to have their child at home.

The third report of this database, figure 3, was about postnatal visits and a query was design for that

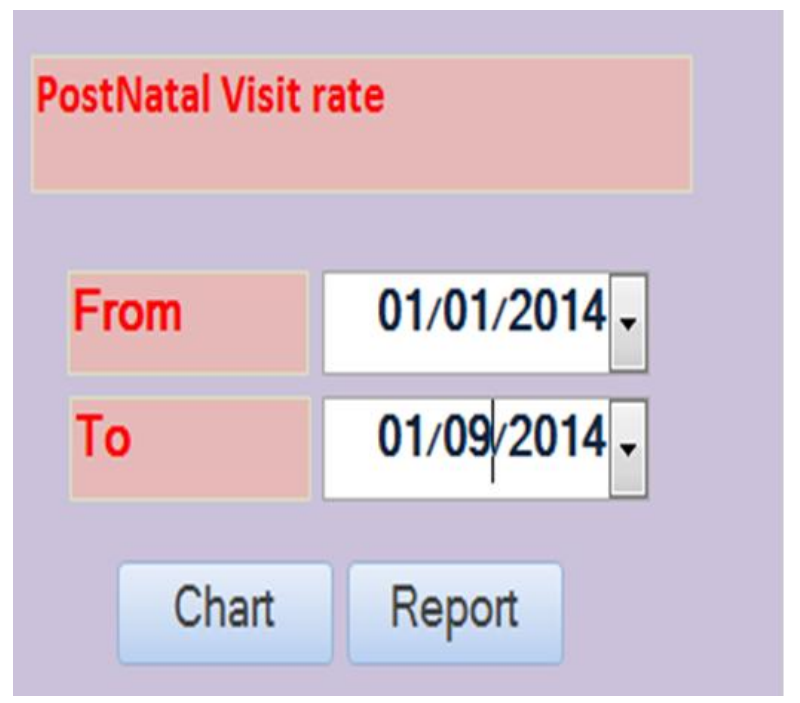

Figure 3: Report about postnatal visits.

Staff's opinion and belief about database were obtained in this study. Their opinion about the feasibility of database was accordingly positive with some concerns. The staff mentioned that the database would help them more easily to do their work. They believed the database was clear and simple in use. The staff mostly mentioned that database would help the decreasing overload as well as save time for them. purpose, while, because of unrecorded data about postnatal care, the database could not show report about postnatal visits. In the same concerning fourth report of this database, figure 4, was assigned for maternal mortality rate, but there was not any record data about maternal death in the program in the health center, therefore database was not able to present a report about maternal mortality rate.

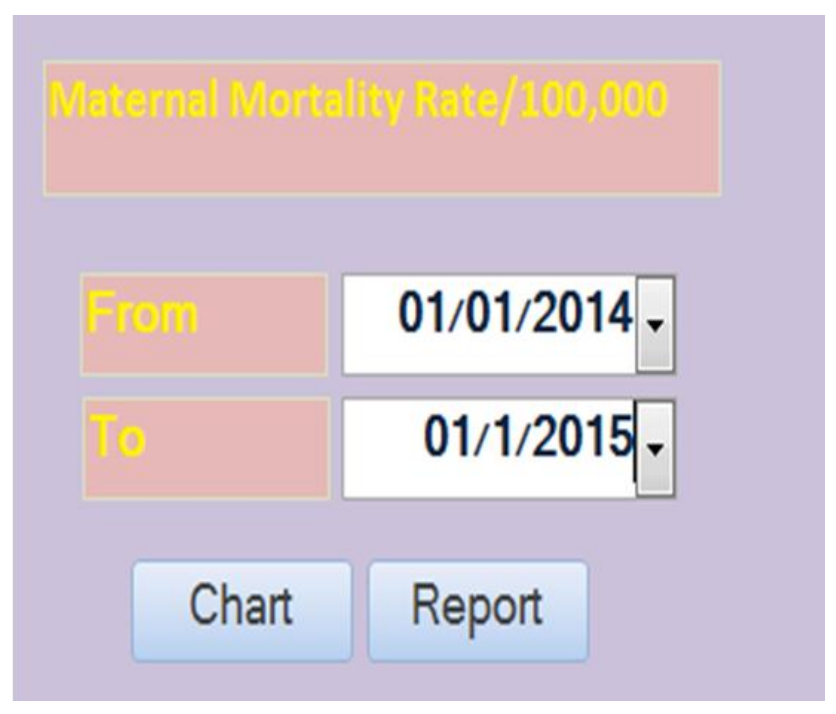

Figure 4: Report about maternal mortality.

Similarly, they believed computerizing mother's file would increase the space of workplace in the room, and all information will be preserved. In addition, search for mother's information in the computer would be easy comparatively, and mother's file could be transferred to another health center. Under this circumstances, some of the staff stated that this database could help them in determining the expected 
number of mothers who supposed to visit the health center in coming day.

In the same way, few of the staff believed practical problems would appear at the real time of performing system. The staff mostly mentioned that, electrical shortage, adequate of computer to in the health center, overload of visited mother, and lack of trained staff would be the main problems when maternal health program used this database. And, few of them declared that low management and logistic support would be another challenge of the computerizing system.

The stakeholder believed that this program has to include the child growth monitoring and immunization to the database, and these departments could connect to each other, in such a way data entry could perform in each department separately. In a same way they suggested that new special department required for data entry. In addition, few of the staff believed there were some services which were so far they were not able to do for the mothers in the maternal health program, such as postnatal care, providing vitamin A for the mothers. These parts could be removed from the database. From another side, there was a suggestion such as connecting the WHO-Anthroplus database to this database (Anthroplus is a database which is launched by WHO for monitoring the growth of children in each area or health center).

The study observed that each mother's form required 10 minute approximately to be entered into database. Another observation for crowd in the health center has illustrated in table 1 . In average the total mothers who visited the health center in a week ( 5 working days) was 134.5. Out of this number 49 of visits were recorded as the first visit and 85.5 of visits were recorded as a regular visit. These figures were not distribute equally for instance, on Saturdays in average 39.5 visits were recorded (11.5 first visits and 28 regular visits), while on Tuesdays were not so much crowd, there was just 16 visits (7.5 first visits and 8.5 regular visits). Tuesdays were assigned to postnatal care visit, but indeed this visit was not for postnatal care, instead of those mothers only have to register their newborn in child monitoring growth department.

Table1. Mother's visiting to the health center in 2 weeks.

\begin{tabular}{|c|c|c|c|c|c|c|}
\hline \multirow[b]{2}{*}{ Days in week } & \multicolumn{3}{|c|}{ Visiting of each days in weeks } & \multicolumn{3}{|c|}{ Average } \\
\hline & $\begin{array}{l}\text { No. First } \\
\text { vising }\end{array}$ & $\begin{array}{l}\text { No. Regular } \\
\text { visiting }\end{array}$ & $\begin{array}{c}\text { Total } \\
\text { visiting }\end{array}$ & $\begin{array}{l}\text { No. First } \\
\text { vising }\end{array}$ & $\begin{array}{l}\text { No. Regular } \\
\text { visiting }\end{array}$ & $\begin{array}{c}\text { Total } \\
\text { visiting }\end{array}$ \\
\hline 2 alternative Sunday & 17 & 33 & 50 & 8.5 & 16.5 & 25 \\
\hline 2 alternative Monday & 17 & 27 & 44 & 8.5 & 13.5 & 22 \\
\hline \multicolumn{7}{|l|}{2 alternative Tuesday } \\
\hline 2 alternative Wednesday & 26 & 38 & 64 & 13 & 19 & 32 \\
\hline 2 alternative Thursday & 15 & 17 & 32 & 7.5 & 8.5 & 16 \\
\hline \multicolumn{7}{|l|}{2 alternative Friday } \\
\hline 2 alternative Saturday & 23 & 56 & 79 & 11.5 & 28 & 39.5 \\
\hline Grand total & 98 & 171 & 269 & 49 & 85.5 & 134.5 \\
\hline
\end{tabular}

\section{Discussion}

This study revealed that making database for maternal health care program had more applicable for monitoring the programs indicators. It also found computerizing program would provide ongoing data about some of those indicators of the program. Antenatal visit and attending health institutions for delivery were the main indicators for monitoring the program in Kurdistan health system. In contrast to that, in this study, this database could not provide data about postnatal visit and maternal mortality indicators. This study observed that the program did not have data about death during antenatal and postnatal period due to maternal causes. Likewise, the program did not give much consider to record about postnatal care. Another reason for that was the program had not been well integrated with child growth monitoring program, because during postnatal period, mothers without any special care directly is referred to child growth monitoring department for the matter of registering her babies. In Tuesday mothers ought to take their file from maternal care department to child growth monitoring department to add their baby's data to the file. Therefore, Tuesday was assigned for registering the newborn in child monitoring growth department as part of their timetable. But, using database would help the staff to work in Tuesdays, because each department could enter their data to computer independently.

From staff prospective, computerizing program would decrease overload, save time and space, and lead to 
better archiving of mother's file. As well as the database would help to easily search for mothers' information, and could better regulate the prenatal and postnatal visits. They believed computerizing the program would better aid integrating maternal health care program with other programs such as child growth monitoring and immunization by making new department to fill all mother's form to computer. On the other hand, health workers also could enter their distinct data in the same local network connected database. They declared that lack of skill in computer use, and shortage in electric would be the main challenges to computerizing process of the program. This database could preserve time when new data is updated during regular visits or second delivery through searching the family code for each mother in the database meanwhile the staff have to use code for searching mother's file manually.

\section{Conclusions and Recommendations}

This database could accelerate the monitoring and evaluating the maternal health program in considering to indicators of the program. Finding from this database and staff opinion illustrate that computerizing the program could better regulate, and integrate the three programs; maternal health care, child growth monitoring and immunization program.

By taking into account the challenges which were addressed in this study, this database could be developed and improved to the maternal and child health program in entire region by the Ministry of Health- Kurdistan Regional Government. In order to the database could provide ongoing data about postnatal visit and maternal mortality, it is essential that the program registers data about mothers death, and integrates postnatal care with child monitoring growth department.

It is recommended that local network connection should be set up in the health center which helps to compromise the E- health system in the health centers of the region.

\section{Limitation of the study}

In this study, we recruit data from one health center because there was only one health center which deliver maternal health program to mothers. In addition we could interview with the few of stakeholders of the program because of same reason. We struggled to expand of our study area to Kalar city near to Darbandikhan, but the health authorities in the region was not cooperate with us.

\section{Acknowledgement}

The authors would like to thank the Darbandikhan administrative of health for their effort and cooperation in this research. We also need to recognize the support of Dr. Sherko, the director of the health in the Darbandikhan to this study

\section{References}

[1] United Nations. Department of Economic and Social Affairs. Population Division 2013. World Population Prospects: The 2012 Revision, DVD Edition

[2] Gilbert Burnham. Perceptions and utilization of primary health care services in Iraq: findings from a national household survey. BMC International Health and Human Rights 2011, $11: 15$.

[3] Therese A Wiegers. The quality of maternity care services as experienced by women in the Netherlands. BMC Pregnancy and Childbirth 2009, 9:18 doi:10.1186/1471-2393-9-18.

[4] WHO. Maternal, child and reproductive health strategy in Iraq 2008

[5] Ala'din Alwan. Health in Iraq: The Current Situation, Our Vision for the Future and Areas of Work. Ministry of health

[6] United Nations. Catharine Way, The Millennium Developing Goals report, United nation-New york, 2015.

[7] WHO. E-Health and innovation in women and children health2013 survey, Atlas of e Health Country Profiles. http://www.who.int/goe)

[8] WHO. Country statistics and global health estimates by WHO and UN parter,2015.

[9] USAID-Iraq. Primary health care: Recording and Reporting of Maternal Deaths in Iraq. October,2012.

[10] USAID Iraq- access: Focused antenatal care: primary integrated, individualized care during pregnancy, February, 2007.

[11] WHO. Conflict and humanitarian crisis in Iraq; Public health risk assessment and interventions, 2014. http://www.who.int/disasters

[12] Nazar P Shabila, Namir G Al-Tawil1, Tariq S Al-Hadithi1, Egbert Sondorp and Kelsey Vaughan. Iraqi primary care system in Kurdistan region: providers' perspectives on problems and opportunities for improvement. BMC International Health and Human Rights 2012, 12:21 http://www.biomedcentral.com/1472-698X/12/21)

[13] WHO. E-Health and innovation in women and children health2013 survey, Atlas of e Health Country Profiles. http://www.who.int/goe).

[14] Nazar P Shabila, Hamdia M Ahmed, and Maryam Y Yasin. Women's views and experiences of antenatal care in Iraq: a Q methodology study BMC Pregnancy Childbirth. 2014; 14: 43: PMCID: PMC3902000 Published online 2014 Jan 23. doi: 10.1186/1471-2393-14-43 